\title{
ISBER 2018 Abstract Category Index
}

\begin{tabular}{|c|c|c|}
\hline Oral Session & $\mathrm{P}-11$ & P-56 \\
\hline & $\mathrm{P}-12$ & P-57 \\
\hline Research into Practice & $\mathrm{P}-13$ & P-58 \\
\hline $\mathrm{O}-01$ & & P-59 \\
\hline $\mathrm{O}-02$ & Biospecimen Research & P-60 \\
\hline $\mathrm{O}-03$ & $\mathrm{P}-14$ & \\
\hline $\mathrm{O}-04$ & $\mathrm{P}-15$ & \\
\hline $\mathrm{O}-05$ & P-16 & Repository Automation Technology \\
\hline O-06 & P-17 & P-61 \\
\hline \multirow[t]{2}{*}{ O-07 } & P-18 & P-62 \\
\hline & P-19 & P-63 \\
\hline Biobanking: Big Opportunities, Big ELSI & P-20 & P-64 \\
\hline $\mathrm{O}-10$ & $\mathrm{P}-21$ & P-99 \\
\hline O-11 & $\begin{array}{l}P-21 \\
P-22\end{array}$ & P-100 \\
\hline O-12 & P-23 & \\
\hline O-14 & P-24 & Repository Management \\
\hline Professional Repository Management & $\mathrm{P}-25$ & $\mathrm{P}-65$ \\
\hline O-15 & P-26 & P-66 \\
\hline $0-16$ & P-27 & P-67 \\
\hline O-17 & $\mathrm{P}-28$ & P-68 \\
\hline O-19 & P-29 & P-69 \\
\hline O-20 & $\mathrm{P}-30$ & P-70 \\
\hline \multirow{2}{*}{$\mathrm{O}-21$} & $\mathrm{P}-31$ & P-71 \\
\hline & $\mathrm{P}-32$ & $\mathrm{P}-72$ \\
\hline Advancing the Norms: & P-33 & $\mathrm{P}-73$ \\
\hline Human Repositories & $\mathrm{P}-34$ & $\mathrm{P}-74$ \\
\hline $\mathrm{O}-22$ & P-35 & P-75 \\
\hline $\mathrm{O}-23$ & P-36 & P-76 \\
\hline $\mathrm{O}-24$ & P-37 & P-77 \\
\hline $\mathrm{O}-25$ & P-38 & P-78 \\
\hline $\mathrm{O}-26$ & P-39 & P-79 \\
\hline $\mathrm{O}-27$ & P-40 & P-80 \\
\hline \multirow{2}{*}{ O-28 } & $\mathrm{P}-41$ & $\mathrm{P}-81$ \\
\hline & & $\mathrm{P}-82$ \\
\hline \multirow{2}{*}{ Poster Session } & Ethical, Legal, and Societal Issues & P-83 \\
\hline & P-42 & P-84 \\
\hline Biobank Tools (for the ISBER Tool & $\mathrm{P}-43$ & $\begin{array}{l}1-84 \\
P-85\end{array}$ \\
\hline Booth) & P-44 & P-86 \\
\hline P-01 & $\mathrm{P}-45$ & $\begin{array}{l}1-00 \\
P-87\end{array}$ \\
\hline \multirow{2}{*}{\multicolumn{3}{|c|}{$\begin{array}{l}\mathrm{P}-\mathrm{U} 2 \\
\mathrm{P}-03\end{array}$}} \\
\hline & & \\
\hline P-04 & Hot Topics & \\
\hline P-101 & $\mathrm{P}-47$ & Repository Standards \\
\hline P-102 & $\mathrm{P}-48$ & P-89 \\
\hline \multirow{2}{*}{ P-103 } & & P-90 \\
\hline & Human Specimen Repositories & P-91 \\
\hline Biobanking Profiles & P-49 & P-92 \\
\hline P-05 & $\mathrm{P}-50$ & P-93 \\
\hline P-06 & P-51 & P-94 \\
\hline P-07 & $\mathrm{P}-52$ & P-95 \\
\hline P-08 & P-53 & P-96 \\
\hline P-09 & $\mathrm{P}-54$ & P-97 \\
\hline P-10 & $\mathrm{P}-55$ & P-98 \\
\hline
\end{tabular}


Innovative Technologies Academic

ITP-1

ITP-2

ITP-3

Innovative Technologies

IT-01

IT-02

IT-03

IT-04

IT-05

IT-06

IT-07

IT-08

IT-09

IT-10

Late Breaking Sessions

Information Technology-LBA

LBP-1

LBP-2

LBP-3

LBP-4

LBP-5

LBP-6

LBP-7

Repository Management-LBA

LBP-8

LBP-9

LBP-10

LBP-11
Biobanking Profiles-LBA

LBP-12

LBP-13

LBP-14

LBP-15

LBP-16

LBP-17

LBP-18

Biospecimen Research and Science-LBA

LBP-19

LBP-20

LBP-21

LBP-22

LBP-23

LBP-24

LBP-25

LBP-26

LBP-27

LBP-28

LBP-29

LBP-30

LBP-31

LBP-32

LBP-33

LBP-34

LBP-35

Biospecimen Research and Science-LUX

LBP-36

LBP-37

LBP-38
Biospecimen Research and ScienceQuality-LBA

LBP-39

LBP-40

LBP-41

LBP-42

Biospecimen Research and ScienceQuality-LUX

\section{LBP-43}

LBP-44

LBP-45

LBP-46

Ethical, Legal, and Societal Issues-LBA LBP-47

LBP-48

LBP-49

LBP-50

LBP-51

Hot Topics-LBA

LBP-52

LBP-53

LBP-54

LBP-55

Human Specimen Repositories-LBA LBP-56

LBP-57

LBP-58

LBP-59

LBP-60 